\title{
Tensor Product Model-based Control Design with Relaxed Stability Conditions for Perching Maneuvers
}

\author{
Yingying Kan ${ }^{1}$, Zhen $\mathrm{He}^{1}$, Jing Zhao ${ }^{2}$ \\ ${ }^{1}$ College of Automation Engineering, Nanjing University of Aeronautics and \\ Astronautics, No.29 Jiangjun Avenue, 211106, Nanjing, China, \\ zdhzsy@nuaa.edu.cn, hezhen@nuaa.edu.cn \\ ${ }^{2}$ School of Automation, Nanjing University of Posts and Telecommunications, \\ No.66 Xin Mofan Road, 210003, Nanjing, China, zhaojing@ njupt.edu.cn
}

\begin{abstract}
This paper proposed a control design approach based on tensor product models for perching maneuvers of fixed-wing aircraft. The highly nonlinear longitudinal dynamics of perching maneuvers is transformed into a tensor product model. The interpolation technique is investigated to reduce the conservatism of the convex tensor product model. The properties of the time derivatives of premise membership functions are utilized in the control design process to further reduce the control conservatism. The proposed method is demonstrated with simulation results.
\end{abstract}

Keywords: perching maneuvers; Tensor product model; Flight control; Parallel distributed compensation control

\section{Introduction}

Compared to rotorcraft, fixed-wing aircraft have the advantages of longer endurance, larger loading capacity and higher flight speed. However, the landing of a conventional fixed-wing aircraft usually needs a large space (e.g. a long runway), which restricts the application of fixed-wing aircraft. In nature, large birds could decelerate rapidly by performing a post-stall maneuver and eventually land on branches. Inspired by this kind of effective landing maneuver of large birds, a new landing approach for fixed-wing aircraft, i.e. the perching maneuver has been proposed and drawn more and more research attentions in recent years [1-5]. In the process of perching maneuver, a fixed-wing aircraft needs to emulate the landing maneuver of large birds, which involves rapidly decelerating by performing a very high angle-of-attack (AoA) flight and landing precisely at a prescribed perch point. 
It is a challenging task to design a controller for fixed-wing aircraft performing perching maneuvers, for the aircraft dynamics is highly nonlinear and time-varying during high AoA maneuver. Studies on the control problem of perching maneuvers are relatively sparse. Stability analysis has been carried out by using contraction theory in [2]. It is shown that deviations in initial state variables will lead to trajectory divergence and prevent the aircraft from reaching the prescribed perch point [2]. In [6], a neighboring optimal control strategy has been used to cope with the perturbations from nominal trajectory. However, the simulation results show that the tolerant perturbations are too narrow [6]. In [5] and [7], a novel control synthesis approach known as LQR-Trees has been used to enlarge allowable perturbed initial conditions. In [2], a controller based on sliding-mode technique has been designed to guarantee successful perching maneuver under perturbed initial conditions.

The tensor product (TP) modeling method is a convenient and effective way to construct TP type polytopic model for nonlinear systems and therefore facilitated control design by enabling convex hull-based algorithms [8-11]. In this paper, the highly nonlinear dynamics of perching maneuvers is transformed into a linear parameter varying (LPV) model and then the corresponding TP modeling is carried out. In order to decrease the conservativeness of control design, the interpolation technique between two types of convex TP models has been investigated and the TP model with less conservativeness has been constructed. Based on the TP model, linear matrix inequality (LMI) stability conditions for the control design have derived and the system stability has been proved. In order to further reduce the conservatism of the controller, the control design process utilized the fuzzy Lyapunov functions and thusthe LMI stability conditions are more relaxed than conventional LMI stability conditions.

The paper is organized as follows: the dynamics and TP modeling of aircraft performing perching maneuvers is presented in Section 2; in Section 3, first a basic control scheme is designed and the conservatism of convex TP model is analyzed by using the interpolation technique; then parallel distributed compensation control design based on fuzzy Lyapunov functions is presented; simulation results are shown in Section 4; finally, the results of the research are concluded.

\section{TP Modeling of Perching Maneuvers}

This paper is focused on the longitudinal motion of perching maneuver, for this high AoA maneuver is mainly related to longitudinal variables. The longitudinal equations of motion (EOM) of fixed-wing aircraft performing perching maneuver are expressed in the wind axes as 


$$
\left\{\begin{array}{l}
\dot{V}=(T \cos \alpha-D-m g \sin \gamma) / m \\
\dot{\gamma}=(T \sin \alpha+L-m g \cos \gamma) / m V \\
\dot{\alpha}=q-(T \sin \alpha+L-m g \cos \gamma) / m V \\
\dot{q}=M / I_{y} \\
\dot{x}=V \cos \mu \\
\dot{h}=V \sin \mu
\end{array}\right.
$$

where $V, \gamma, \alpha, q$ denote flight velocity, flight-path angle, angle of attack and pitch angle rate respectively; $x$ and $h$ represent horizontal displacement and altitude of the aircraft; $m$ is the aircraft mass and $I_{y}$ is the moment of inertia; $T$ is the thrust; the lift $L$ and the drag $D$ are aerodynamic forces, and $M$ is the aerodynamic moment.

The aerodynamic forces of the aircraft in the longitudinal plane can be written as

$$
\left\{\begin{array}{l}
L=\frac{1}{2} \rho V^{2} S C_{L} \\
D=\frac{1}{2} \rho V^{2} S C_{D}
\end{array}\right.
$$

where $C_{L}$ and $C_{D}$ denote the lift and drag coefficients of the aircraft respectively. $\rho$ is the air density and $S$ is the aerodynamic surface area of the aircraft. The aerodynamic behavior of aircraft during perching maneuvers is highly nonlinear. Combining the experimental and analytic results in $[1,2]$ and the plate model theory mentioned in [12], in this work the aerodynamic coefficients are given as follows:

$$
\left\{\begin{array}{l}
C_{L}=0.8 \sin (2 \alpha) \\
C_{D}=1.4(\sin \alpha)^{2}+0.1
\end{array}\right.
$$

Assume that the aircraft uses an all-moving horizontal tail, so that it can acquire relatively large control moment even at low flight velocity. Then, the pitching moment is calculated as

$$
M=-\frac{1}{2} \rho V^{2} S_{e} l_{e}\left(\begin{array}{l}
0.8 \cos \alpha \sin \left(2 \alpha+2 \delta_{e}\right)+ \\
1.4 \sin \alpha \sin ^{2}\left(\alpha+\delta_{e}\right)+0.1 \sin \alpha
\end{array}\right)
$$

where $\delta_{e}$ is the deflection angle of elevator, $S_{e}$ represents the aerodynamic surface area of elevator and $l_{e}$ denotes the distance from the aerodynamic center of elevator to the mass center of aircraft. 
Let $\boldsymbol{X}=[V, \gamma, \alpha, q]^{T}$ and $\boldsymbol{u}=\left[T, \delta_{e}\right]^{T}$ and substitute the aerodynamic terms (2)-(4) into the equations of motion (1). Then the equations of motion (1) can be rearranged into two groups of equations as follows:

$$
\begin{gathered}
\dot{\boldsymbol{X}}=\boldsymbol{f}(\boldsymbol{X}, \boldsymbol{u}) \\
\left\{\begin{array}{l}
\dot{x}=V \cos \gamma \\
\dot{h}=V \sin \gamma
\end{array}\right.
\end{gathered}
$$

where $f(\square)$ is a nonlinear function vector, the specific form of which can be easily obtained from (1)-(4) and is not presented here for brevity.

The reference trajectory is assumed to be specified priori and described by $\left(\boldsymbol{X}_{r}, \boldsymbol{u}_{r}\right)$, where $\boldsymbol{X}_{r}=\left[V_{r}, \gamma_{r}, \alpha_{r}, q_{r}\right]^{T}$ and $\boldsymbol{u}_{r}=\left[T_{r}, \delta_{e r}\right]^{T}$, which satisfy $\dot{\boldsymbol{X}}_{r}=\boldsymbol{f}\left(\boldsymbol{X}_{r}, \boldsymbol{u}_{r}\right)$. Along the reference trajectory, the displacement variables $x_{r}$ and $h_{r}$ satisfy $\left\{\begin{array}{l}\dot{x}_{r}=V_{r} \cos \gamma_{r} \\ \dot{h}_{r}=V_{r} \sin \gamma_{r}\end{array}\right.$.

Then the control problem of perching maneuver is often treated as a trajectory tracking control problem, which means that a flight controller needs to be designed to make the actual states of the aircraft $\boldsymbol{X}$ and $(x, h)$ to track the reference states $\boldsymbol{X}_{r}$ and $\left(x_{r}, h_{r}\right)$ respectively.

Linearizing the nonlinear dynamics (5) along the reference trajectory and omitting the higher order terms would yield a LPV model, the system matrices of which are denoted as $A$ and $B . A$ and $B$ depend on exogenous parameters $\left(\boldsymbol{X}_{r}, \boldsymbol{u}_{r}\right)$. However, since $\left(\boldsymbol{X}_{r}, \boldsymbol{u}_{r}\right)$ consists of six variables, it would be arduous to directly construct TP model. Considering that the variables in $\left(\boldsymbol{X}_{r}, \boldsymbol{u}_{r}\right)$ are all functions of time, thus in the paper we express the system matrices $A$ and $B$ as functions of time and then we could construct the TP model by choosing the transformation space as $t \in\left[a_{t}, b_{t}\right]$. Thus the corresponding linearized model is as follows:

$$
\Delta \dot{\boldsymbol{X}}_{r}=\left.\frac{\partial \boldsymbol{f}}{\partial \boldsymbol{X}}\right|_{\substack{\boldsymbol{X}=\boldsymbol{X}_{r} \\
\boldsymbol{u}=\boldsymbol{u}_{r}}} \Delta \boldsymbol{X}_{r}+\left.\frac{\partial \boldsymbol{f}}{\partial \boldsymbol{u}}\right|_{\substack{\boldsymbol{X}=\boldsymbol{X}_{r} \\
\boldsymbol{u}=\boldsymbol{u}_{r}}} \Delta \boldsymbol{u}_{r}=\left[\begin{array}{ll}
\boldsymbol{A}(t) & \boldsymbol{B}(t)
\end{array}\right]\left[\begin{array}{c}
\Delta \boldsymbol{X}_{r} \\
\Delta \boldsymbol{u}_{r}
\end{array}\right]
$$


where $\quad \Delta \boldsymbol{X}_{r}=\boldsymbol{X}-\boldsymbol{X}_{r} \quad, \quad \Delta \boldsymbol{u}_{r}=\boldsymbol{u}-\boldsymbol{u}_{r} \quad, \quad \boldsymbol{A}(t)=\left.\frac{\partial \boldsymbol{f}}{\partial \boldsymbol{X}}\right|_{\substack{\boldsymbol{X}=\boldsymbol{X}_{r} \\ \boldsymbol{u}=\boldsymbol{u}_{r}}} \quad$ and $\boldsymbol{B}(t)=\left.\frac{\partial \boldsymbol{f}}{\partial \boldsymbol{u}}\right|_{\substack{\boldsymbol{X}=\boldsymbol{X}_{r} \\ \boldsymbol{u}=\boldsymbol{u}_{r}}}$. The parameter matrices $\left.\frac{\partial \boldsymbol{f}}{\partial \boldsymbol{X}}\right|_{\substack{\boldsymbol{X}=\boldsymbol{X}_{r} \\ \boldsymbol{u}=\boldsymbol{u}_{r}}}$ and $\left.\frac{\partial \boldsymbol{f}}{\partial \boldsymbol{u}}\right|_{\substack{\boldsymbol{X}=\boldsymbol{X}_{r} \\ \boldsymbol{u}=\boldsymbol{u}_{r}}}$ in the dynamics (7) depend on the variables along the reference trajectory and hence are time-varying.

Next, the TP modeling method is applied to (7) to construct the TP type ploytopic model of the perching maneuver. For the model (7), define the transformation space as $t \in\left[a_{t}, b_{t}\right]$. Discretize the space by a grid with the size of $M$, where $M$ is a integer. Then the TP model transformation can be executed by using the TPtool Matlab toolbox [13]. The detailed procedure of this transformation method has been elaborated in the literatures [8-11]. The time-varying matrices in (7) can be approximated by a convex combination of linear-time-invariant (LTI) matrices, that is

$$
\left[\begin{array}{ll}
\boldsymbol{A}(t) & \boldsymbol{B}(t)
\end{array}\right]=\sum_{i_{t}=1}^{J} w_{t, i_{t}}(t) \times\left[\begin{array}{ll}
\boldsymbol{A}_{i_{t}} & \boldsymbol{B}_{i_{t}}
\end{array}\right]
$$

where $\boldsymbol{A}_{i_{t}}$ and $\boldsymbol{B}_{i_{t}}$ are constant matrices; $w_{t, i_{t}}$ are weighting functions satisfying the following convex criteria [8]:

$$
\left\{\begin{array}{l}
\forall i_{t}, t: w_{t, i_{t}}(t) \in[0,1] \\
\forall i_{t}, t: \sum_{i_{t}=1}^{J} w_{t, i_{t}}(t)=1
\end{array}\right.
$$

\section{Control Design with Relaxed Stability Conditions}

\subsection{Basic Controller Design and Conservatism Analysis of Convex TP Models}

Before studying the conservatism reduction of the TP models, a basic controller needs to be designed to ensure the stability of the closed-loop system. Then, the conservatism of different TP models can be compared through the solvability of 
the design conditions and the control performances. The structure of the basic controller is shown in Figure 1.

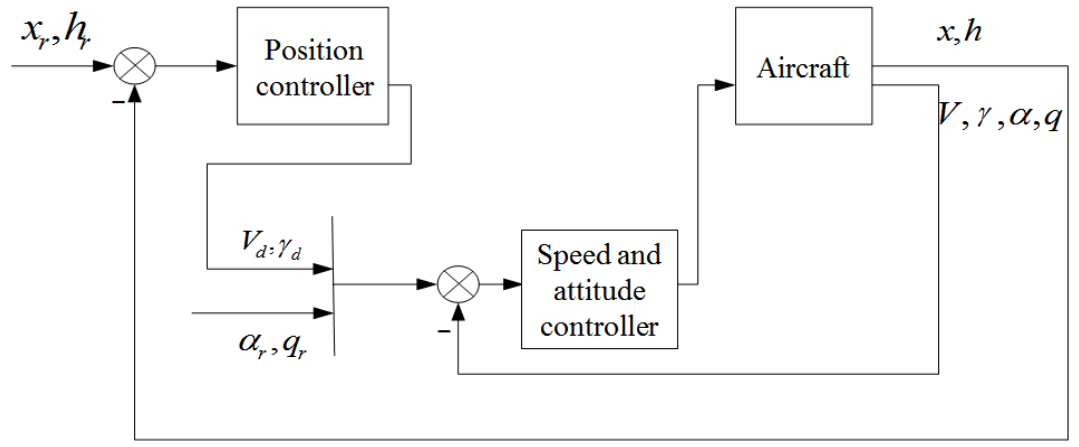

Figure 1

The basic controller structure

From (6) it can be known that the position tracking error satisfies the following relationship:

$\left\{\begin{array}{l}\Delta \dot{x}=V \cos \gamma-V_{r} \cos \gamma_{r} \\ \Delta \dot{h}=V \sin \gamma-V_{r} \sin \gamma_{r}\end{array}\right.$

where $\Delta x=x-x_{r}, \Delta h=h-h_{r}$. Suppose that the position tracking error is controlled by a proportional controller as

$\left\{\begin{array}{l}\Delta \dot{x}=k_{1} \Delta x \\ \Delta \dot{h}=k_{2} \Delta h\end{array}\right.$

However, the dynamics (11) cannot be implemented directly, for the actual flight speed $V$ and the flight-path angle $\gamma$ in (10) cannot responsed to commands immediately. Considering that the dynamics of position is slower than the flight speed and the flight-path angle, it is reasonable to utilize the flight speed and the flight-path determined from (10) and (11) (which are denoted as $V_{d}$ and $\gamma_{d}$ respectively) as the reference values for the actual speed and the flight-path angle. $V_{d}$ and $\gamma_{d}$ are calculated by

$$
\left\{\begin{array}{l}
V_{d}=\sqrt{\left(V_{r} \cos \mu_{r}+k_{1} \Delta x\right)^{2}+\left(V_{r} \sin \mu_{r}+k_{2} \Delta h\right)^{2}} \\
\gamma_{d}=\arctan \left(\frac{V_{r} \sin \mu_{r}+k_{2} \Delta h}{V_{r} \cos \mu_{r}+k_{1} \Delta x}\right)
\end{array}\right.
$$


Denote $\boldsymbol{X}_{d}=\left[V_{d}, \gamma_{d}, \alpha_{r}, q_{r}\right]$ and $\Delta \boldsymbol{X}_{d}=\boldsymbol{X}-\boldsymbol{X}_{d}$.Then from (7) and (8), the dynamics of $\Delta \boldsymbol{X}_{d}$ is obtained as

$$
\Delta \dot{\boldsymbol{X}}_{d}=\sum_{j=1}^{J} w_{j}\left[\begin{array}{ll}
\boldsymbol{A}_{j} & \boldsymbol{B}_{j}
\end{array}\right]\left[\begin{array}{l}
\Delta \boldsymbol{X}_{d} \\
\Delta \boldsymbol{u}_{r}
\end{array}\right]
$$

Note that the terms containing $\left(\boldsymbol{X}_{r}-\boldsymbol{X}_{d}\right)$ and its derivative in the dynamics of $\Delta \boldsymbol{X}_{d}$ are neglected in (13). The speed and attitude controller is designed according to the parallel distributed compensation control scheme as follows:

$$
\Delta \boldsymbol{u}_{r}=\sum_{j=1}^{J} w_{j} \boldsymbol{K}_{j} \Delta \boldsymbol{X}_{d}
$$

Substituting (14) into (13) yields

$$
\Delta \dot{\boldsymbol{X}}_{d}=\sum_{k=1}^{J} \sum_{j=1}^{J} w_{j} w_{k}\left(\boldsymbol{A}_{j}+\boldsymbol{B}_{j} \boldsymbol{K}_{k}\right) \Delta \boldsymbol{X}_{d}
$$

The closed-loop TP model (15) can be rewritten into a compact form as

$$
\Delta \dot{\boldsymbol{X}}_{d}=S(p(t)) \Delta \boldsymbol{X}_{d}
$$

where $S(p(t))=\mathrm{S})^{N} w_{n}\left(p_{n}(t)\right)$ is the tensor expression of the coefficient matrices. Different ty ${ }^{n} \bar{p}$ es of convex weight functions can be chosen to derive TP model. Proper weight function type would decrease the conservatism of the system [11, 14-18]. Here the interpolation technique [11] is studied to obtain a TP model with less conservatism. The convex weight function types CNO and SNNN are considered and the corresponding convex hull expressions are as follows:

$$
\begin{aligned}
& \left.S(p(t))={ }^{C N O} \mathrm{~S}\right)_{n=1}^{N}{ }^{C N O} w_{n}\left(p_{n}(t)\right) \\
& \left.S(p(t))={ }^{S N N N} \mathrm{~S}\right)_{n=1}^{N}{ }^{S N N N} w_{n}\left(p_{n}(t)\right)
\end{aligned}
$$

The discrete weighting functions of the two convex hulls as shown in (17) and (18) are interpolated as

$$
{ }^{I(\xi)} w_{n}^{D(\Omega, \mathrm{G})}=(1-\xi)^{S N N N} w_{n}^{D(\Omega, \mathrm{G})}+\xi^{C N O} w_{n}^{D(\Omega, \mathrm{G})}
$$

where $\xi \in[0,1]$ is the interpolation coefficient and the superscript $D$ means discrete weight function. 
Then by using the pseudo tensor product model transformation, the interpolated convex hull can be obtained as

$$
\left.S(p(t))={ }^{I(\xi)} \mathrm{S}\right)_{n=1}^{N}{ }^{I(\xi)} w_{n}\left(p_{n}(t)\right)
$$

The gains of the parallel distributed compensation controller (14) need to be designed so that the closed-loop system is stable. Define a common Lyapunov function as $V=\Delta X_{d}{ }^{\mathrm{T}} P \Delta X_{d}$. The following LMIs can be derived to ensure the stability of the closed-loop system:

$$
\begin{aligned}
& X A_{j}^{T}+A_{j} X+M_{j}^{T} B_{j}^{T}+B_{j} M_{j}<0 \\
& X A_{j}^{T}+A_{j} X+X A_{k}^{T}+A_{k} X+M_{k}^{T} B_{j}^{T}+B_{j} M_{k}+M_{j}^{T} B_{k}^{T}+B_{k} M_{j} \leq 0, j<k
\end{aligned}
$$

where $k=1, \ldots, J, X$ is a positive definite symmetric matrix and $X=P^{-1}$, and $M_{k}$ is a matrix with appropriate dimensions. The control gains are calculated as $K_{k}=M_{k} X^{-1}$.

In addition, pole placement techniques can be used to allocate closed-loop poles [19]. Define a pole region $\ell(\tau)=\{s \mid \operatorname{Re}(\mathrm{s})<-\tau<0\}$ in the complex plane. The following LMIs guarantee that the closed-loop poles are located in region $\ell(\tau)$

$$
A_{j} X+X^{T} A_{j}^{T}+B_{j} M_{k}+M_{k}^{T} B_{j}^{T}+2 \tau X<0, j, k=1, \ldots, J
$$

Solving the LMIs (21) and (22), the control gains in (14) can be determined as $K_{k}=M_{k} X^{-1}$, which guarantee both the stability and the pole location of the system. The maximum $\tau$ (which is denoted as $\tau_{\max }$ ) of a closed-loop system can be determined by iteratively solving the LMIs (21) and (22).

The conservatism of the convex hull (20) under different interpolation coefficients is analyzed by comparing the solvability of the above LMIs and $\tau_{\max }$. The relationship between the interpolation coefficient $\xi \in[0,1]$ and $\tau_{\max }$ is depicted in Figure 2. In Figure 2, the zero value of $\tau_{\max }$ means that the LMIs are not solvable in this case. 


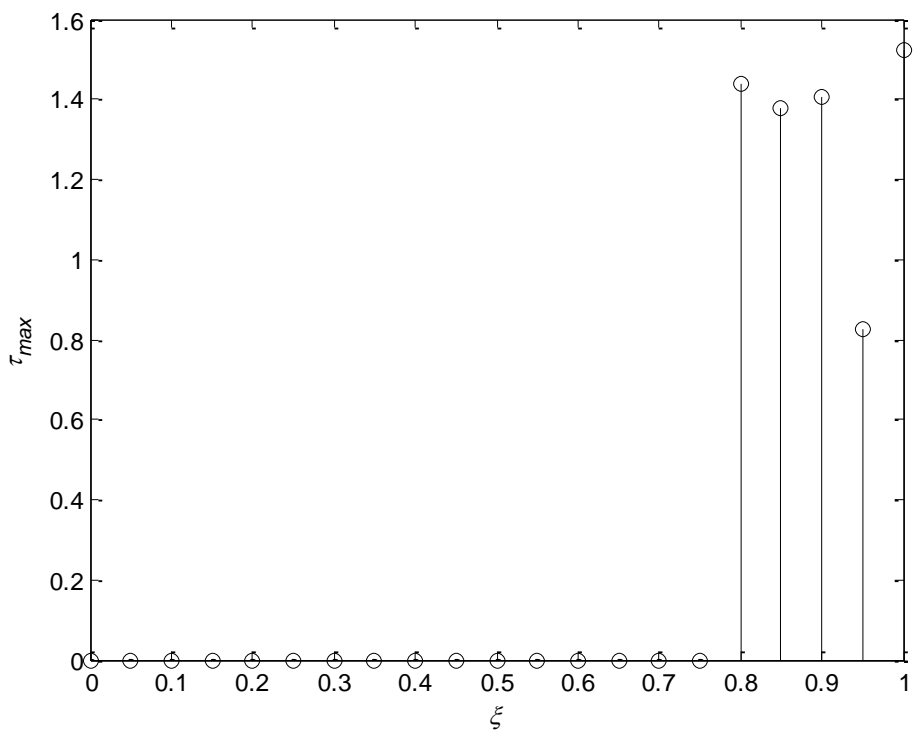

Figure 2

The relationship between $\xi$ and $\tau_{\max }$

It can be seen from Figure 2 that the solvability of the LMIs are influenced by the interpolation coefficient $\xi$. When $\xi$ is in the range of $[0.8,1]$, the LMIs $(21)$ and (22) are solvable and the controller (14) can be obtained. In addition, $\tau_{\text {max }}$ also varies with $\xi$. The larger $\tau_{\max }$ is, the faster the system responses. Thus, it is beneficial to choose the value of $\xi$ corresponding to relatively large $\tau_{\max }$ in the range of $[0.8,1]$. It is reasonable to think that the convex hull (20) under this chosen interpolation coefficient is less conservative.

\subsection{Control Design Based on Fuzzy Lyapunov Functions}

In section 3.1, the parallel distributed compensation controller (14) is designed by using common Lyapunov functions. The control design conditions (21) and (22) are derived in this way. However, control design based on common Lyapunov functions usually has strong conservatism. In order to further reduce the conservatism of the system, in this section the control design is based on fuzzy Lyapunov functions. By using fuzzy Lyapunov functions the properties of the time derivatives of premise membership functions can be exploited and therefore the LMI stability conditions can be relaxed [20-22].

The stability conditions for the perching maneuvers are presented in the following. Consider the close-loop system (15). Assume that $\left|\dot{w}_{\rho}\right| \leq \phi_{\rho}$, where $\phi_{\rho}$ is a positive real number and $\rho=1, \ldots, J$. The closed-loop system is asymptotically stable if for some fixed $i=1, \ldots, J$ and a scalar $\varepsilon>0$ there exist positive definite 
symmetric matrices $S, Q_{\rho}$ and matrices $Z, Y_{k},(k=1, \ldots, J)$ satisfying the following LMIs:

$$
\begin{aligned}
& Q_{\rho}>0, \forall \rho \in\{1, \ldots, J\} \\
& Q_{\rho}+S-Q_{i} \geq 0, \forall \rho \in\{1, \ldots, J\}-\{i\} \\
& {\left[\begin{array}{ll}
\tilde{Q}_{\phi}-A_{j} Z-Z^{T} A_{j}^{T}-B_{j} Y_{j}-Y_{j}^{T} B_{j}^{T} & * \\
Q_{j}-\varepsilon\left(A_{j} Z+B_{j} Y_{j}\right)+Z^{T} & \varepsilon\left(Z+Z^{T}\right)
\end{array}\right]<0, j=1, \ldots, J} \\
& {\left[\begin{array}{ll}
\tilde{Q}_{\phi}-A_{j} Z-Z^{T} A_{j}^{T}-B_{j} Y_{k}-Y_{k}^{T} B_{j}^{T} & * \\
Q_{j}-\varepsilon\left(A_{j} Z+B_{j} Y_{k}\right)+Z^{T} & \varepsilon\left(Z+Z^{T}\right)
\end{array}\right]+\left[\begin{array}{ll}
\tilde{Q}_{\phi}-A_{k} Z-Z^{T} A_{k}^{T}-B_{k} Y_{j}-Y_{j}^{T} B_{k}^{T} & * \\
Q_{k}-\varepsilon\left(A_{k} Z+B_{k} Y_{j}\right)+Z^{T} & \varepsilon\left(Z+Z^{T}\right)
\end{array}\right]<0} \\
& j<k, j, k=1, \ldots, J
\end{aligned}
$$

where $\tilde{Q}_{\phi}= \pm \phi_{i} S+\sum_{\substack{\rho=1 \\ \rho \neq i}}^{J} \phi_{\rho}\left(Q_{\rho}+S-Q_{i}\right)$ with the symbol \pm indicating that the signs + and - must be tested.

The local gains can be obtained as $K_{k}=Y_{k} Z^{-1}$. The proof of LMI stability conditions (23)-(26) can be directly derived according to [22]. In LMIs (23)-(26), the values of $i, \varepsilon$ and $\phi_{\rho}$ would influence the resulting control gains and need to be determined according to practical situation.

The controller designed by solving the LMIs (23)-(26) can guarantee the stability of the TP model. Consider that the approximation accuracy of TP model (15) is relatively high, and the time-varying model (7) can approximate the nonlinear model with relatively high accuracy if the aircraft operates near the reference trajectory. Thus, the designed controller is effective for the aircraft (described by the nonlinear model) if the aircraft operates near the reference trajectory.

\section{Simulation Case}

Simulations are carried out to demonstrate the effectiveness of the proposed control design method. Consider a fixed-wing aircraft with the main parameters as follows: $m=0.8 \mathrm{~kg}, \quad I_{y}=0.1 \mathrm{~kg} \cdot \mathrm{m}^{2}, \quad S=0.25 \mathrm{~m}^{2}$ and $S_{e}=0.054 \mathrm{~m}^{2}$. 
Considering the practical requirement that the thrust weight ratio of fixed-wing aircraft is less than 1 and the thrust should not below zero, the actuator constraint of the aircraft is set as $T \in[0,7.8 \mathrm{~N}]$. The transformation space of TP modeling is chosen as $t \in[0,1.6 \mathrm{~s}]$. Discretize the space by a grid with the size of $M=100$. Then the TP model transformation can be executed by using the TPtool Matlab toolbox [13]. There are 12 nonzero singular values, the four biggest ones of which are kept. The weight functions are shown in Figure 3.

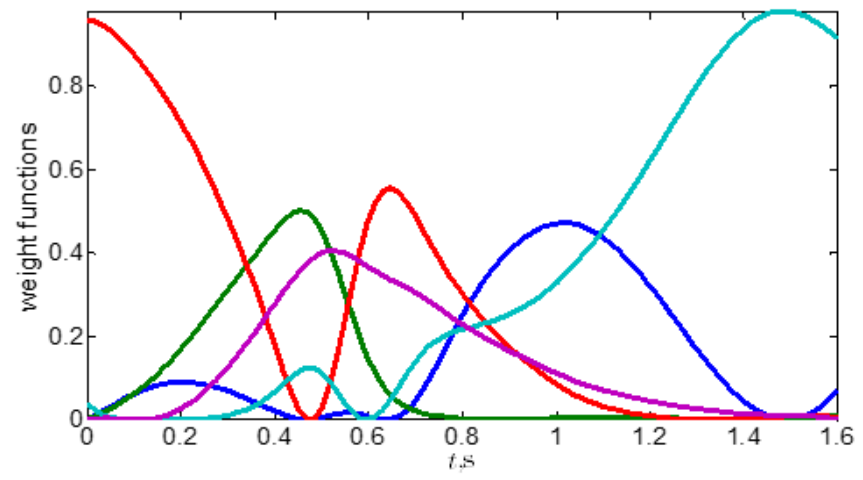

Figure 3

Weight functions

The position control gains in (11) are chosen as $k_{1}=-2, k_{2}=-8$. For the LMIs (23)-(26), the values of $i$ and $\varepsilon$ are chosen as $i=5$ and $\varepsilon=0.1$. The suprema of the time derivatives of premise membership functions are specified as:

$$
\phi_{1}=2.1696, \phi_{2}=3.3704, \phi_{3}=5.2277, \phi_{4}=1.8306, \phi_{5}=1.6080
$$

Then solve the LMIs (23)-(26) for the control gains in (14).

The initial reference states of the aircraft are set as: $V=9.9736 \mathrm{~m} / \mathrm{s}, \mu=0$, $\alpha=0.2455 \mathrm{rad}, q=0, x=0$ and $h=0$. Two kinds of simulation cases are carried out. In case 1 , the initial deviation of flight speed is set as $-1 \mathrm{~m} / \mathrm{s}$, while in case 2 , the initial deviation of flight speed is set as $1 \mathrm{~m} / \mathrm{s}$. The initial error of angle of attack is set as $1^{\circ}$.

In order to demonstrate the benefit of the proposed control scheme, the simulation results of the controller designed based on fuzzy Lyapunov function (the proposed control scheme) are compared with the simulation results of the controller designed based on common Lyapunov function.

First, consider the simulation case 1 . The response curves of the state variables are shown in Figure 4. It can be seen from Figure 3 that, for both kinds of controllers, the angle of attack increases rapidly and the flight speed decreases from $9 \mathrm{~m} / \mathrm{s}$ to about $4 \mathrm{~m} / \mathrm{s}$, which is relatively slow. Figure 5 shows the trajectory history of the aircraft. For both kinds of controllers, the actual trajectories can track the reference trajectory and the final position error is relatively small and acceptable 
considering the size of the aircraft. However, as shown in Figure 6, the thrust command generated by the controller based on common Lyapunov function exceeds the constraint $T \in[0,7.8 \mathrm{~N}]$. Nevertheless, Figure 6 also shows that, for the controller based on fuzzy Lyapunov function, though the thrust and the elevator deflection do not converge to their reference curves, they are all kept within reasonable ranges. To some extent, this demonstrates that the proposed control design scheme for perching maneuvers is less conservative than the control design approach based on common Lyapunov functions. Thus, the simulation results demonstrate the effectiveness and benefit of the proposed controller.

The results of simulation case 2 are shown in Figure 7. It can be seen from Figure 7 that, similarly to the simulation case 1 , the control command generated by the proposed controller is relatively mild, while the thrust command generated by the controller based on common Lyapunov function exceeds its constraint (in this case the thrust command is less than zero at the beginning period), though the response curves of the two kinds of controllers are similar. This verifies the benefit of using the proposed controller.
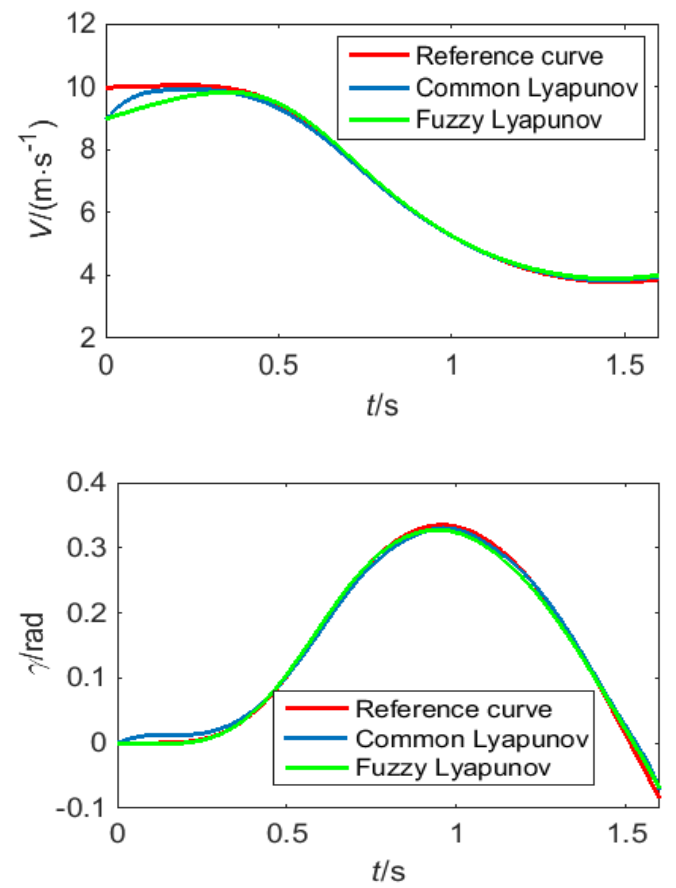

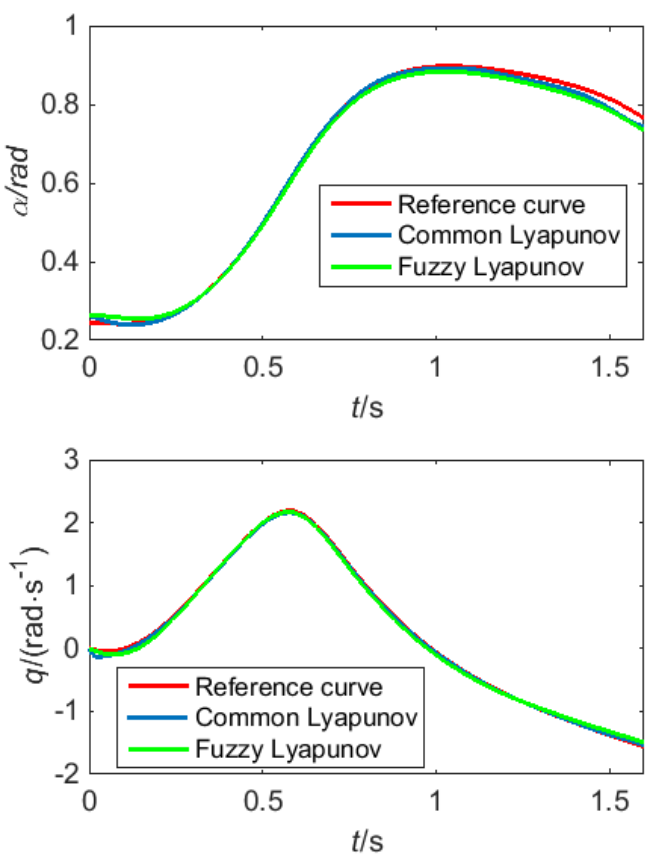

Figure 4

Response curves of the state variables in case 1

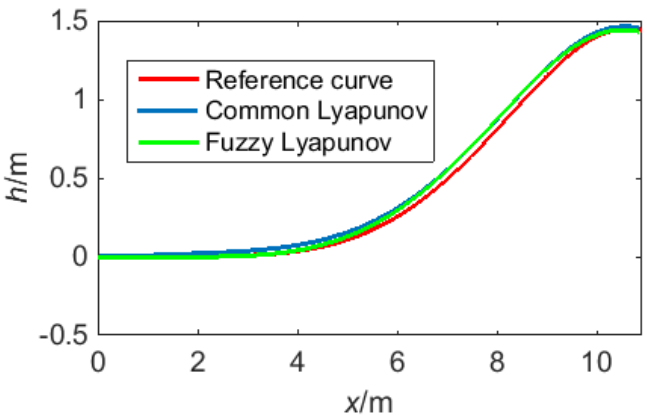

Figure 5

Trajectory history in case 1 

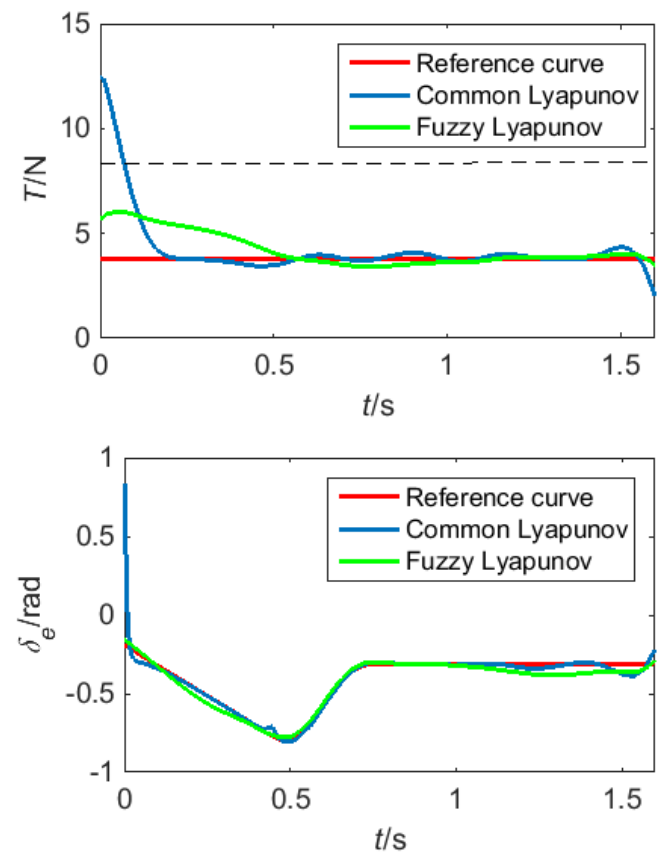

Figure 6

Control inputs in case 1
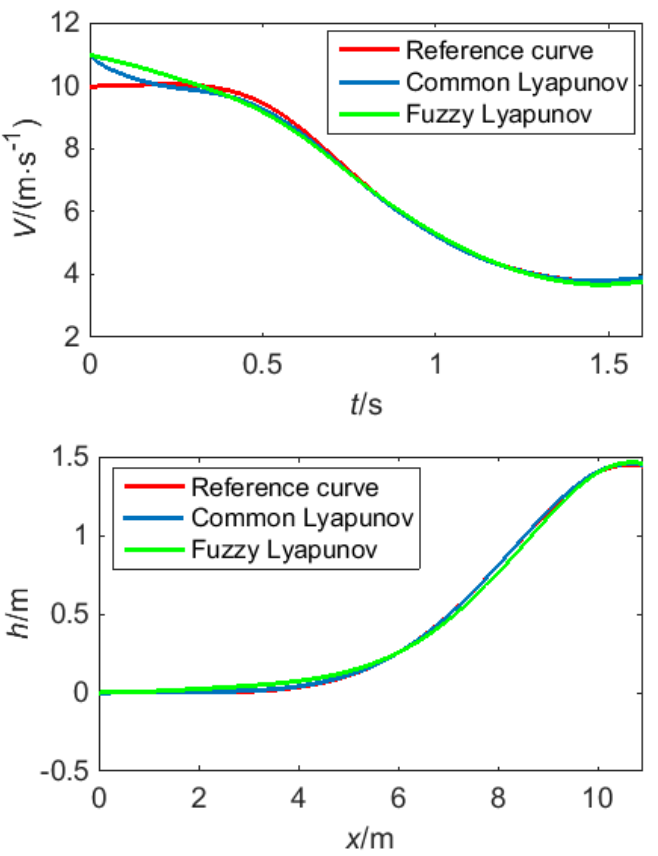

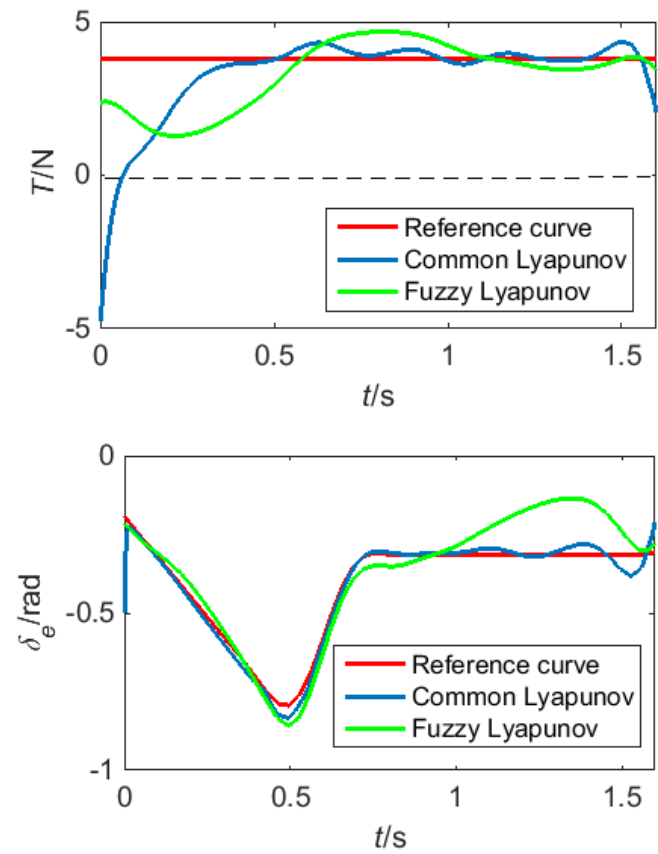

Figure 7

Response curves and inputs in case 2

\section{Conclusions}

In this paper, a tensor product model-based control design method is investigated for aircraft performing perching maneuvers. In order to decrease the conservativeness of control design, the interpolation technique between two types of convex TP models has been investigated and the TP model with less conservativeness has been constructed. The control design is carried out by using fuzzy Lyapunov functions, and the properties of the time derivatives of premise membership functions are considered in the design process. Therefore, the resulting LMI stability conditions are more relaxed than conventional LMI stability conditions. Simulation results show that the proposed control approach can ensure successful perching maneuvers, which demonstrates the effectiveness of the control design method. In addition, the simulation results of the proposed controller and the controller based on common Lyapunov function are compared, which demonstrates that the proposed controller is less conservative.

\section{Acknowledgement}

This work was supported by the National Natural Science Foundation of China (No. 61304139, 61403210). 


\section{References}

[1] D. M. K. K. Venkateswara Rao, H. Tang, and T. H. Go, A parametric study of fixed-wing aircraft perching maneuvers, Aerospace Science and Technology, 42: 459-469, 2015

[2] D. M. K. K. Venkateswara Rao, and T. H. Go, Optimization, stability analysis, and trajectory tracking of perching maneuvers, Journal of Guidance, Control, and Dynamics, 37(3): 879-888, 2014

[3] M. AliKhan, N. K. Peyada, and T. H.Go, Flight dynamics and optimization of three-dimensional perching maneuver, Journal of Guidance, Control, and Dynamics, 36(6): 1791-1797, 2013

[4] S. Regisford, and J. VanderMey, Perching a Minimally-Actuated Micro Air Vehicle, 51 ${ }^{\text {st }}$ AIAA Aerospace Sciences Meeting, Grapevine (Dallas/Ft. Worth Region), AIAA Paper 2013-0359, Jan. 2013

[5] J. L. Moore, Robust post-stall perching with a fixed-wing UAV, Doctoral dissertation, Massachusetts Institute of Technology, 2014

[6] S. Regisford, and Parry, An investigation into the use of neighboring optimal control in the solution of a perching problem, $51^{\text {st }}$ AIAA Aerospace Sciences Meeting including the New Horizons Forum and Aerospace Exposition, Grapevine(Dallas/Ft. Worth Region), AIAA Paper 2013-0766, Jan. 2013

[7] J. Moore, R. Cory, and R. Tedrake, Robust post-stall perching with a simple fixed-wing glider using LQR-Trees, Bioinspiration \& biomimetics, 9(2): $1-15,2014$

[8] P. Baranyi, TP model transformation as a manipulation tool for QLPV analysis and design. Asian Journal of Control, 17: 497-507, 2015

[9] B. Takarics and P. Baranyi, Tensor-product-model-based control of a three degrees-of-freedom aeroelastic model. Journal of Guidance, Control, and Dynamics, 36: 1527-1533, 2013

[10] P. Baranyi and B. Takarics, Aeroelastic wing section control via relaxed tensor product model transformation framework. Journal of Guidance, Control, and Dynamics, 37: 1671-1678, 2014

[11] P. Baranyi, Y. Yam and P. Várlaki, Tensor product model transformation in polytopic model-based control. Boca Raton, Florida: CRC Press, 2013

[12] R. E.Cory, Supermaneuverable perching, Doctoral dissertation, Massachusetts Institute of Technology, 2010

[13] TPtool MATLAB Toolbox. Natick: MathWorks, MA, 2007 
[14] P. Baranyi, The Generalized TP model transformation for T-S fuzzy model manipulation and generalized stability verification. Fuzzy Systems, IEEE Transactions on, 22: 934-948, 2014

[15] P. Baranyi, TP-Model transformation-based-control design frameworks. Springer International Publishing Switzerland, 2016 (doi: 10.1007/978-3-319-19605-3)

[16] A. Szollosi and P. Baranyi, Influence of the tensor product model representation of QLPV models on the feasibility of linear matrix inequality. Asian Journal of Control, 18(4): 1328-1342, 2016

[17] A. Szollosi and P. Baranyi, Improved control performance of the 3-DoF aeroelastic wing section: a TP model based 2D parametric control performance optimization. Asian Journal of Control, 19(2): 450-466, 2017

[18] A Szollosi and P. Baranyi, Influence of the tensor product model representation of qLPV models on the feasibility of linear matrix inequality based stability analysis, Asian Journal of Control, 2017 in print

[19] M. Chilali and P. Gahinet, $\mathrm{H}_{\infty}$ design with pole placement constraints: a LMI approach. IEEE Transactions on Automatic Control, 41: 358-367, 1996

[20] J. T. Pan, T. M. Guerra, S. M. Fei, et al. Nonquadratic stabilization of continuous T-S fuzzy models: LMI solution for a local approach. IEEE Transactions on Fuzzy Systems, 20(20):594-602, 2012

[21] B. J. Rhee, S. Won, A new fuzzy Lyapunov function approach for a Takagi--Sugeno fuzzy control system design. Fuzzy Sets \& Systems, 157(9):1211-1228, 2006

[22] F. A. Faria, G. N. Silva, V. A. Oliveira, Reducing the conservatism of LMI-based stabilisation conditions for TS fuzzy systems using fuzzy Lyapunov functions. International Journal of Systems Science, 44(10): 1956-1969, 2013 